\title{
Jejunal Injury as a Rare Complication of Percutaneous Nephrolithotomy: Case Report
}

Khaled M. Al-Kohlany, MD, Khaled A. Telha, MD, Noman Al-lahabi, MD, and Hani M. Almahmoud, MSc

\begin{abstract}
Background: Colonic injury represents a rare complication of percutaneous nephrolithotomy (PCNL). Injury of the small bowel is very rare, with only anecdotal case reports in the literature. We report here a rare PCNL complication of jejunal perforation without concomitant colonic injury.

Case Presentation: A 45-year-old male underwent PCNL for multiple left kidney stones. At the beginning of the procedure, we faced difficulty in achieving access, but after that the procedure was straightforward and ended smoothly. However, from the third day, the postoperative course became stormy with progressive development of picture of acute peritonitis. Laparotomy was mandatory and jejunal perforation was found and repaired.

Conclusion: Although extremely rare, small bowel injury should be kept in mind during planning for percutaneous nephrolithotomy. Prompt diagnosis and active treatment should be undertaken without delay especially if picture of acute peritonitis is developed.
\end{abstract}

Keywords: percutaneous nephrolithotomy, complications, bowel injury

\section{Introduction and Background}

B OWEL INJURIES ARE rare complications of percutaneous nephrolithotomy (PCNL), representing about $0.2 \%$ to $0.8 \%$ of all PCNL complications. In the majority of cases, the colon is the bowel affected by the injury, especially in certain circumstances. ${ }^{1}$ Small bowels are usually away from the PCNL pathway and thus are protected from injuries during PCNL. Nevertheless, few reports are available in the literature about small bowel injuries, including ileum ${ }^{2}$ and jejunum. ${ }^{3}$ Herein, we report about rarely observed jejunal injury during PCNL that progressed as acute peritonitis and required laparotomy. In addition, we tried to explain the mechanism of this injury and reviewed the literature about this rare complication and its management.

\section{Case Presentation}

A 45-year-old healthy male presented with left loin pain of several months' duration. After proper evaluation, he was diagnosed as having multiple stones in the left kidney. He had irrelevant previous medical and surgical history and had unremarkable clinical examination. He had normal laboratory investigations, including sterile urine culture. Urinary tract plain and intravenous urography showed left renal pelvic and lower calix stones $2.5 \mathrm{~cm}$ in maximum length without hydronephrosis (Fig. 1a, b). He was scheduled for elective PCNL. Because of intrarenal pelvis, a stone casting the renal pelvis and lower calix, absence of hydronephrosis, and more dependent lower calix with vertical caudal orientation, difficult access to the kidney was expected and consent for conversion to open surgery was obtained.

We perform PCNL by the classic technique, starting by cystoscopic insertion of ureteral stent in lithotomy position. After that the patient is positioned in prone position with elevation of the affected side, about $30^{\circ}$, by a pillow. Contrast is injected through the ureteral catheter and the collecting system is opacified. Under fluoroscopic guidance, we usually chose to access the kidney by monoplanar technique with the fluoroscope only in vertical position, relying on the movement of the kidney and retraction of the targeted calix. In

Department of Urology, General Military Hospital and Medical College, Sana'a University, Sanaa, Yemen.

(c) Khaled M. Al-Kohlany et al. 2017; Published by Mary Ann Liebert, Inc. This is an Open Access article distributed under the terms of the Creative Commons Attribution License, which permits unrestricted use, distribution, and reproduction in any medium, provided the original work is properly cited. Mary Ann Liebert, Inc. offers reprint services for those who want to order professionally produced copies of articles published under the Creative Commons Attribution (CC BY) license. To obtain a price quote, email Reprints@liebertpub.com. Please include the article's title or DOI, quantity, and delivery destination in your email. 

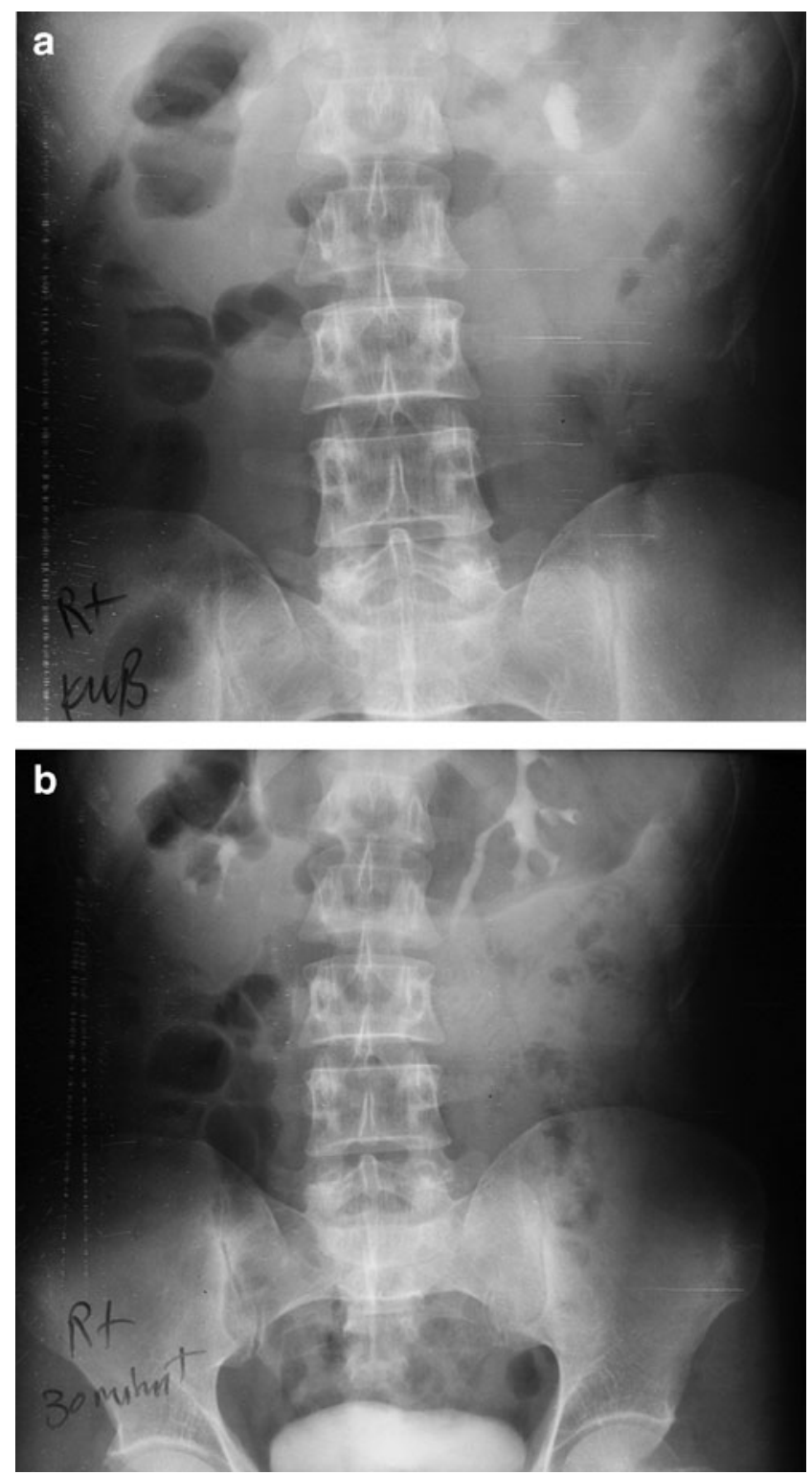

FIG. 1. (a) KUB showing two radio-opaque shadows representing the two stones in the left renal region. (b) Intravenous urography showing the location of the stones in the collecting system and the configuration of the pelvicaliceal system. KUB, kidney, ureter, and bladder radiograph.

most of the cases, we chose the lower or middle posterior calix for access according to the collecting system anatomy and stone distribution. Rarely, we use the upper calix for puncture. In most of the cases, we use only one super stiff guidewire over which central Alken's rod is inserted. Then, dilatation of the tract is performed by Alken's metallic coaxial dilators, over which 30-32F Amplatz sheath is introduced. Pneumatic lithoclast and ultrasound are used for stone disintegration. By the end of procedure, if there is collecting system injury, bleeding, or significant residual stone, antegrade Double- $J$ stent is inserted and nephrostomy tube is left. If the procedure proceeds smoothly without residual stones or collecting system injury, we only leave an external ureteral stent and do not leave nephrostomy tube (tubeless PCNL).
In our current case, there was difficulty in puncturing the kidney at the beginning of the procedure, but on fourth trial, access was achieved and the tract was dilated. The procedure after that passed smoothly and the stones were cleared, antegrade Double-J stent was placed, and nephrostomy tube (PCN) was left behind because of pelvicaliceal system laceration inflicted during tract dilatation.

The patient was asymptomatic on postoperative days 1 and 2 and therefore was given fluids and progressed to regular diet. On the third day, he developed high-grade fever, abdominal distention, and pain. Complete blood count was normal. However, bowel injury was suspected and nasogastric tube was inserted and parenteral IV fluids started. On the fourth day, the condition deteriorated more and we observed yellowish watery discharge coming beside the PCN with fecal odor. The abdominal pain and tenderness increased significantly. The patient was planned for antegrade nephrostogram study and contrast CT scan study of the abdomen, but after surgical consultation, the decision was for urgent laparotomy when erect plain abdomen X-ray showed multiple air-fluid levels and air under diaphragm (Fig. 2).

Laparotomy was done on the same day. This showed peritonitis with spillage of fecal material on the left side and pelvis. There was a jejunal loop entrapped between the descending colon below the splenic flexure and the nephrostomy tube (PCN). After its mobilization, it was found to be partially injured with perforation of about 1/4th of the circumference (Fig. 3). The descending colon was found posterior to the lower pole of the kidney and was intact, but compressed by the nephrostomy tube, which was passing

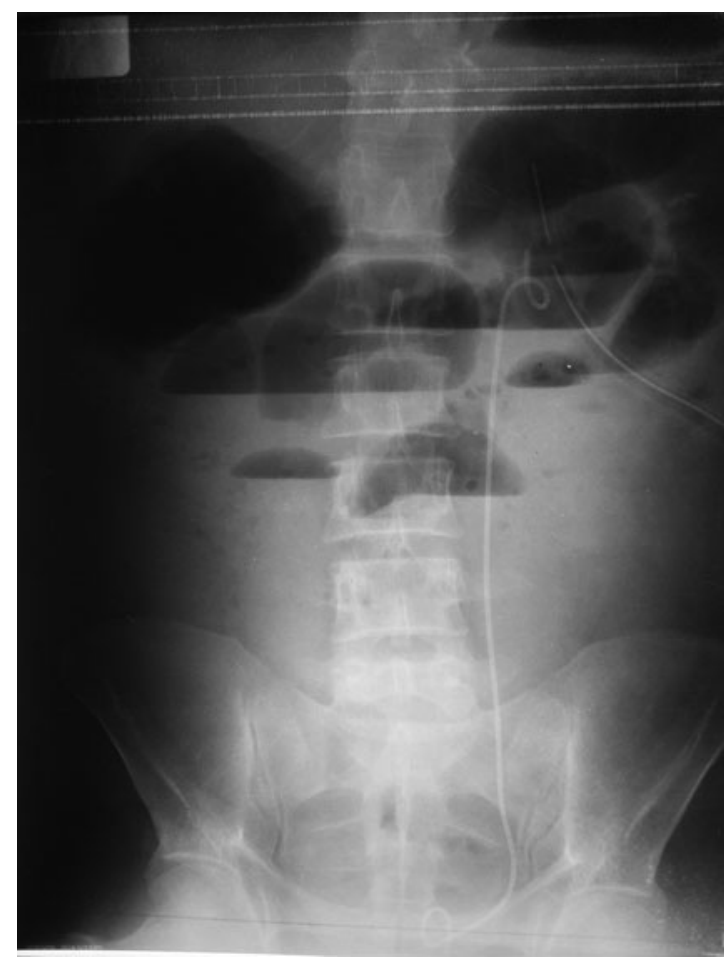

FIG. 2. Erect abdominal plain film showing the multiple air-fluid levels and air under diaphragm. 


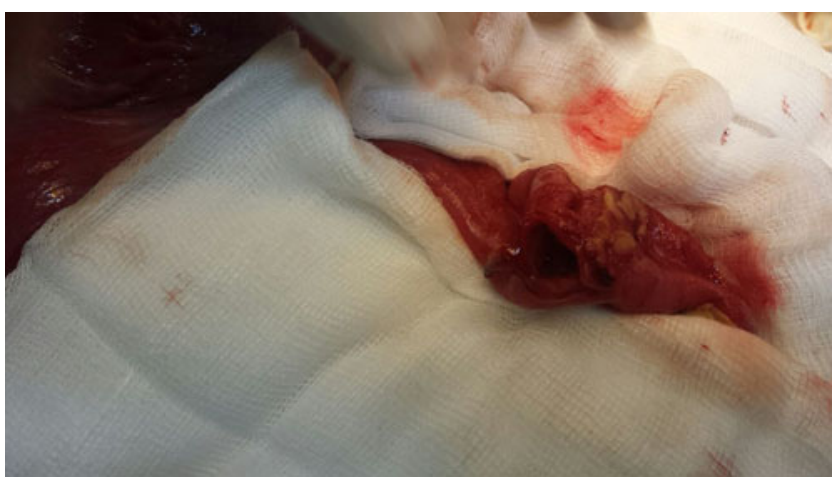

FIG. 3. The jejunal perforation: close view of the perforation showing the size of the injury related to the jejunal circumference.

over it without any injury and entering the kidney through the lower calix. The nephrostomy tube was removed, the jejunum was repaired, and peritoneal lavage was performed. The patient passed smooth postoperative course, resumed normal bowel habits on the third day, started oral fluids on the fourth day, and was discharged home on the seventh day.

\section{Discussion}

Bowel injuries are one of the rarest PCNL complications with incidence ranging from $0.2 \%$ to $0.8 \% .^{1}$ Because of its rarity, the available reports in the literature are scarce and are usually case reports. Because of their location and close relation to the kidneys, the ascending and descending colon are the most involved parts of the bowels by this complication. Several risk factors have been proposed for this complication, including renal and or colonic abnormalities. Retrorenal position of the colon, which is found in as many as $15 \%$ of patients, has been implicated as a risk factor for this complication. ${ }^{4}$ Small bowels are usually not along the path of the renal tract dilatation during PCNL and therefore are seldom injured unless an intra-abdominal entry is sustained. There are only isolated reports of jejunal and ileal injuries in literature. ${ }^{2,3}$

Some institutions recommend the routine use of $\mathrm{CT}$ in planning for percutaneous surgery to detect any anatomical abnormalities of the kidneys, to determine their relationships with neighboring organs, to assess the stone burden, and to plan for proper percutaneous access. Tuttle and colleagues reported about the importance of using multiplanar reformatted $\mathrm{CT}$ in prone and supine positions rather than on prone axial CT only to assess the risk of injury of adjacent organs during percutaneous nephrostomy access. ${ }^{4}$ Although it is a useful tool, unfortunately, we cannot perform this study in our hospital as routine for all cases because of economic and organization reasons. However, in certain cases when there is high suspicion of anatomic problem of the kidneys or the stone burden is not clear on conventional imaging, we request it and plan our procedure accordingly. In fact, in our case, preoperatively we did not have any doubt about the abnormal relationship of the kidney to the bowels, so we did not perform CT scan.
After extensive literature search, we found only one report about jejunal injury. Al-Assiri and colleagues reported jejunal injury during left PCNL in an 83-year-old male patient. $^{3}$ They managed this complication conservatively with favorable outcome. In our case, we suspected colonic injury and were planning for conservative management. However, progressive clinical deterioration of the patient's condition changed our plan to urgent abdominal exploration where we found jejunal perforation, which explained the stormy clinical picture.

In retrospect, in trying to understand and explain the mechanism of this unusual complication in this particular patient, we found several factors that might be speculated as possible explanation. First of all, the collecting system configuration with the lower calix harboring the stone is more dependent and with caudal direction. Second, upon reviewing the patient's history, he mentioned that he had chronic constipation with bowel evacuation every 3-4 days. Upon reviewing the X-rays of the patient, there was some sort of bowel distention before surgery. The distal colon was probably evacuated by the routine rectal enema given on the night of surgery while the small bowels remained distended.

Third, the stones were casting the calix and the renal pelvis, thus making more resistance during puncture and dilatation and pushing the kidney more medially toward the bowels. Fourth, the puncture needle and the tract dilators passed from retroperitoneum over the colon through the peritoneal cavity to reach the more vertical lower calix. In their way, they tangentially perforated the jejunum without entering its lumen and continued their way to the kidney. This was unrecognized during surgery and as the jejunum was perforated and freely draining inside the peritoneal cavity, picture of acute peritonitis progressively developed especially with early return to regular oral diet from the second postoperative day.

Finally, the conservative treatment usually recommended for colonic injuries during PCNL and described for the reported case with jejunal injury had no place in our case due to clinical deterioration of the patient's condition.

Although before surgery we were expecting difficult access for PCNL and obtained consent for possible conversion to open surgery, the relatively smooth course of the procedure relieved our concerns. Nevertheless, in retrospect and after getting this serious complication, we realized that our concern was right and more care and evaluation should be undertaken before and during intervention. In this regard, revision of the technique with change of the approach to the kidney possibly through an upper calix approach, passing the guidewire into the ureter, and the use of a single-step balloon dilatation may be employed to avoid an intra-abdominal injury such as this.

\section{Conclusion}

Despite its extremely rare incidence, small bowel injury still represents serious possible complication that should be kept in mind while planning for PCNL. More attention should be paid for patients with lower caliceal stones or those with abnormal collecting system configuration, which makes access difficult. Prompt evaluation and adequate decision for the management of this serious complication 
should be properly decided, including major surgical intervention.

\section{Acknowledgment}

The authors would like to thank their colleague Dr. Noman Al-lahabi from surgical department for his assistance in surgical management of this patient.

\section{Disclosure Statement}

No competing financial interests exist.

\section{References}

1. Michel MS, Trojan L, Rassweiler JJ. Complications in percutaneous nephrolithotomy. Eur Urol 2007;51:899-906.

2. Saad KSM, Hanno A, El-Nahas AR. Injury of the ileum during percutaneous nephrolithotomy in a pediatric patient. Can Urol Assoc J 2014;8:204-206.

3. Al-Assiri M, Binsaleh S, Libman J, Anidjar M. Jejunal perforation during percutaneous nephrolithotripsy. Scientific World J 2005;5:496-499.

4. Tuttle DN, Yeh BM, Meng MV, et al. Risk of injury to adjacent organs with lower pole fluoroscopically guided percutaneous nephrostomy: Evaluation with prone, supine and multiplanar reformmated CT. J Vasc Interv Radiol 2005; 16:1489-1492.
Address correspondence to: Khaled M. Al-Kohlany, MD Department of Urology General Military Hospital and Medical College Sana'a University P.O. Box: 314 Altahreer Post Office

Sanaa

Yemen

E-mail: kalkohlani@gmail.com; kalkohlani@yahoo.com

$$
\begin{aligned}
& \text { Abbreviations Used } \\
& \mathrm{CT}=\text { computed tomography } \\
& \mathrm{PCNL}=\text { percutaneous nephrolithotomy }
\end{aligned}
$$

Cite this article as: Al-Kohlany KM, Telha KA, Al-lahabi N, Almahmoud HM (2017) Jejunal injury as a rare complication of percutaneous nephrolithotomy: case report, Journal of Endourology Case Reports 3:1, 70-73, DOI: $10.1089 /$ cren.2017.0025. 